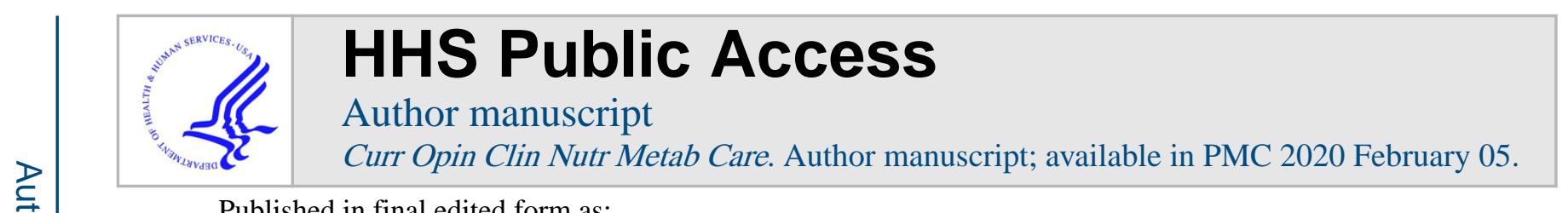

Published in final edited form as:

Curr Opin Clin Nutr Metab Care. 2018 July ; 21(4): 267-272. doi:10.1097/MCO.0000000000000480.

\title{
The Manifold Role of the Mitochondria in Skeletal Muscle Insulin Resistance
}

\author{
W. Todd Cade, PT, PhD \\ Program in Physical Therapy \& Department of Medicine, Washington University School of \\ Medicine, Campus Box 8502, 4444 Forest Park Avenue, St. Louis, MO 63108
}

\section{Summary}

The pathophysiology of skeletal muscle insulin resistance is likely multifactorial involving many coordinated physiological processes. However, it is apparent that the mitochondria play an essential role in skeletal muscle insulin sensitivity in health, aging and in numerous metabolic diseases. Deciphering the manifold functions of the mitochondria will allow us to understand the complex relationship between mitochondria and skeletal muscle insulin resistance.

\section{Keywords}

Mitochondria; muscle; lipid; reactive oxygen species; signaling

\section{Introduction}

Approximately 100 million adults, or almost 1/3 of the US population, have either type 2 diabetes or prediabetes; conditions characterized by insulin resistance[1]. Insulin resistance is a condition of decreased responsiveness to insulin (i.e. insulin action) of peripheral tissues including skeletal muscle, liver and adipose tissue. However, due to the large role of skeletal muscle in mediating peripheral glucose uptake in response to insulin (>80\%), skeletal muscle thought to be the main contributor to the development of insulin resistance in humans[2].

Identifying the mechanisms for insulin resistance in the development of type 2 diabetes has been vigorously pursued for decades. The role skeletal muscle mitochondria plays in the development of insulin resistance has been particularly an area of intense investigation and debate[3, 4]). Mitochondria are organelles involved in many different cellular functions including energy production, cellular signaling, heme production, $\mathrm{Ca}^{2+}$ regulation, apoptosis, and autophagy (reviewed in [5]). The knowledge surrounding the role the mitochondria plays in insulin resistance has significantly advanced over the past 20 years. The purpose of this paper is to review the most recent evidence and current thought regarding the role of the mitochondria in skeletal muscle insulin resistance.

Tel: +1 314286 1432; Fax: +1 314286 1410; tcade@wustl.edu.

Conflicts of interest

None declared. 


\section{Mitochondrial 'deficiency', lipid accumulation and insulin resistance}

Over 50 years ago, Randle and colleagues reported that byproducts (acetyl-CoA, NADH, ATP) of fatty acid oxidation can induce skeletal muscle insulin resistance through pyruvate dehydrogenase inhibition, subsequently termed the 'Randle hypothesis' [6]. Although some tenets of this hypothesis have been challenged[7], numerous studies have since found a strong relationship between fatty acid metabolism and insulin resistance (reviewed in [8]). Several studies using a high-fat diet model in rodents have shown that increasing fatty acid levels lowers mitochondrial biogenesis (e.g. PGC-1a) and enzyme capacity (reviewed in [3]). Also, obesity, physical inactivity, and the accumulation of lipid in non-adipose tissues has been strongly associated with insulin resistance in humans [9] (also reviewed in [3]). A series of key studies found lower skeletal muscle mitochondrial oxidative enzyme capacity and morphological alterations (i.e. smaller mitochondria) in obese and diabetic participants compared to healthy, lean individuals. In addition, these studies also found accompanying higher skeletal muscle lipid content and lower insulin sensitivity in these obese and diabetic individuals (reviewed in $[3,4]$ ). Other investigators reported impaired mitochondrial and fatty acid oxidative capacity in insulin resistant individuals (reviewed in [10]). Moreover, concurrent improvements in skeletal muscle oxidative capacity, fat oxidation and insulin sensitivity in conjunction with a reduction in skeletal muscle lipid content with exercise training in obese individuals[11] further suggested that increasing mitochondrial oxidative capacity and fat metabolism decreases skeletal muscle lipid content and improves insulin sensitivity. Later evidence found that fatty acid metabolites, specifically fatty acid CoA, diacylglyerol and ceramides, were strongly associated with disrupted insulin signaling in skeletal muscle (reviewed in [7]). Altogether, these findings pointed to mitochondrial deficiency as the cause of impaired fatty acid oxidation capacity and skeletal muscle fat accumulation, ultimately leading to the mitochondrial driven "lipotoxicity" hypothesis of insulin resistance[12].

Although a strong relationship between altered skeletal muscle lipid metabolism and insulin resistance was established, not all studies and thought agreed that mitochondrial deficiency caused lipid-induced insulin resistance. For example, Holloszy[4] highlighted that although mitochondrial capacity is lower in type 2 diabetic muscle, due to the large capacity of skeletal muscle mitochondria to increase oxidative capacity during exercise, reductions in oxidative capacity in insulin resistant muscle should not have any effect on the basal capacity to oxidize fatty acids and thus cannot be the cause of excess skeletal muscle lipid accumulation. Other studies demonstrated that increased fatty acid flux in skeletal muscle during high-fat diets either had either no effect or in contrast increased mitochondrial biogenesis and capacity. Transgenic models upregulating mitochondrial biogenesis targets (i.e. PGC-1a, PPAR- $a$ ) have shown increased mitochondrial oxidative protein content and capacity paradoxically have shown increased susceptibility to diet-induced insulin resistance (all reviewed in [13]). In humans, Fisher-Wellman et al. recently found normal mitochondrial capacity and content in obese, insulin resistant individuals[14]. In a notable and well-publicized study, Goodpaster et al.[15] found that endurance athletes had elevated skeletal muscle lipid content and high mitochondrial capacity; a condition termed the 'athlete's paradox'. Lastly, a recent study where inducible pluripotent stem cells were generated from genetically insulin resistant humans found that insulin resistance might 
induce mitochondrial dysfunction-not the reverse[16]. Taken together, these studies strongly refute the notion that mitochondrial deficiency causes skeletal muscle insulin resistance.

\section{Skeletal muscle mitochondrial bioenergetics}

Many of these early studies neglected the role mitochondrial bioenergetics plays in interpretation of the mitochondria-substrate metabolism relationship in skeletal muscle. This idea was highlighted by Neufer and colleagues $([13,17])$ in two eloquent perspective papers. In these papers, the authors suggested that mitochondrial fuel load (carbon flux through $\beta$ oxidation), production of reactive oxygen species (ROS), and the resultant inter-relationships with the redox balance more likely causes skeletal muscle insulin sensitivity rather than impaired mitochondrial capacity. In other words, in obesity and during high-fat feeding, fuel supply consistently outpaces energy demands resulting in mitochondrial oxidant production and emission which can result in impaired insulin signaling and insulin resistance[17]. The chemiosmotic theory states that ATP production is contingent on the reduction potential of the electron transport chain accepting electrons from reducing equivalents (e.g. NADH, $\mathrm{FADH}_{2}$ ) generated from the Kreb's cycle and the transference (i.e. pumping) of the donated protons into the intermembrane space that ultimately generates the electric, or membrane potential necessary for ATP synthase activation. In the condition of low energy demands (i.e. rest, low ATP resynthesis), that a 'back pressure' of protons on the outer surface of the inner mitochondrial membrane opposes additional pumping of protons from reducing equivalents and slows electron flow and $\mathrm{O}_{2}$ consumption[17]. Because the inner mitochondrial membrane is inherently 'leaky' to electrons (which generates ROS), electron flow and $\mathrm{O}_{2}$ consumption continues at a basal or 'idling' rate (state 2 respiration). In the conditions of increase carbon flux (i.e. obesity, high-fat diet) and elevated redox state $\left(\uparrow \mathrm{NADH} / \mathrm{NAD}^{+}, \uparrow\right.$ FADH2/FAD ${ }^{+},\left(\uparrow \mathrm{NADPH} / \mathrm{NADP}^{+}\right)$and in the absence of high-energy demand (i.e. exercise), there is a constant state of 'back pressure'. This results in incomplete $\beta$-oxidation, intermediate production and elevated mitochondrial inner membrane electron leak and ROS generation. This is important because several intermediates and byproducts of $\beta$-oxidation, redox and ROS pathways have been shown to be potent primary and secondary signaling mechanisms[5].

Two recent articles have suggested a potential mechanism underlying the relationship between skeletal muscle mitochondrial bioenergetics and insulin resistance. First, Sparks et al.[18•] discovered that gene expression of the mitochondrial inner membrane adenine nucleotide translocator 1 (ANT1), a mitochondrial ATP for cytosolic ADP exchanger, was elevated in exercise-trained subjects compared to untrained subjects and increased ANT1 expression was associated with increased fatty acid-induced uncoupling and insulin sensitivity. Morrow et al.[19••] subsequently found that mitochondrial capacity was decreased and uncoupling and proliferation were increased in ANT1-deficient mice, and that these mice were resistant to high-fat diet-induced lipotoxicity and insulin resistance. Although these findings appear contradictory, both studies used models where energy consumption was increased (e.g. either through exercise or uncoupling). This is an important concept was highlighted by Muoio and Neufer[13] as with increased energy consumption, redox pressure ('back pressure') is relieved, potentially resulting in lower deleterious ROS and $\beta$-oxidation intermediate generation. 


\section{$\beta$-oxidation}

$\beta$-oxidation is the process of fatty acid catabolism to acetyl-CoA which subsequently enters the Kreb's cycle for the generation of reducing equivalents (e.g. NADH, $\mathrm{FADH}_{2}$ ) that enter the electron transport chain. During lipid overload, a constant substrate flux through $\beta$ oxidation ensues and in the presence of incomplete $\beta$-oxidation, produces short- and longchain intermediates called acylcarnitines which may play a role in mitochondrial acyl-CoA balance and glucose homoestasis[20]. A recent publication by Aguer et al.[21] in differentiated $\mathrm{C} 2 \mathrm{C} 12$, primary mouse, and human myotubes that were treated with acylcarnitines (C4:0, C14:0, C16:0) or with palmitate found a reduction in insulin sensitivity in both conditions and that palmitate-induced insulin resistance was rescued by inhibiting acylcarnitine production. Both conditions of insulin resistance were associated with an increase in oxidative stress levels indicating that excess acylcarnitines might contribute to skeletal muscle insulin resistance through ROS signaling. Another recent mouse study[22] and several recent human studies have confirmed this relationship between acylcarnitines and insulin resistance. In humans, Sun et al. • found in a large longitudinal study $(n>2,000)$ that plasma acylcarnitines were strongly predictive of the development of type 2 diabetes[23]. Batchuluun et al. (2018) also found acylcarntines predicted the development of type 2 diabetes but in this study, following gestational diabetes[24]. Lastly, Consitt et al.[25] found a reduced response in insulin-stimulated acylcarnitine lowering in older individuals that was accompanied by a blunting of acetyl-CoA carboxylase phosphorylation; identifying a potential mechanism for acylcarnitine-mediated metabolic inflexibility and insulin resistance. Together, increased generation of acylcarnitines during times of lipid overload appear to contribute to skeletal muscle insulin resistance.

\section{Reactive oxygen species and redox signaling}

Much of the recent data on the relationship between mitochondria and insulin resistance focuses on reactive oxygen species and redox signaling. A large body of evidence suggests that excess mitochondrial ROS production is associated with insulin resistance[26]. An elevated redox state can generate excess ROS which includes the superoxide anion radical $\left(\mathrm{O}_{2}{ }^{\bullet-}\right)$, hydrogen peroxide $\left(\mathrm{H}_{2} \mathrm{O}_{2}\right)$ and the highly reactive hydroxyl radial $\left({ }^{\circ} \mathrm{OH}\right) . \mathrm{H}_{2} \mathrm{O}_{2}$, has been shown to be the primary signaling ROS generated by the mitochondria[27] and an increased production of $\mathrm{H}_{2} \mathrm{O}_{2}$ seems to play a significant role in skeletal muscle insulin resistance[28]. A recent study by Konopka et al. $\bullet$ in obese humans supported this by demonstrating that exercise training can reduce mitochondrial $\mathrm{H}_{2} \mathrm{O}_{2}$ emissions, improve mitochondrial efficiency and restore insulin sensitivity[29]. Another recent study suggested that reducing $\mathrm{H}_{2} \mathrm{O}_{2}$ by increasing antioxidant capacity might improve insulin sensitivity through reducing of intramuscular lipid accumulation rather than a reduction in ROS signaling[30].

$\mathrm{H}_{2} \mathrm{O}_{2}$ appears to be involved in the regulation of several redox signaling pathways including $\mathrm{NADPH} / \mathrm{NADP}^{+}$and the glutathione (GSH/GSSG) and thioredoxin $\left(\operatorname{Trx}_{\mathrm{Red}} / \operatorname{Trx}_{\mathrm{Ox}}\right)$ redox couples (reviewed in [17,31]). Evidence suggests that the redox environment regulates protein phosphorylation/dephosphorylation through redox-sensitive sulfur containing Cys residues found in most mammalian phosphatase enzymes[32]. This is potentially important in the mediation of skeletal muscle insulin resistance as the insulin receptor substrate (IRS) 
has approximately $70 \mathrm{Ser} / \mathrm{Thr}$ residues that are subject to phosphorylation. Also, alterations in Ser/Thr phosphatase tone can also affect the kinetic activity of Ser/Thr kinases; known effectors of skeletal muscle insulin resistance[17, 33]. Recent studies have shown the important role of Ser/Thr protein kinase 25 (STK25) in diet induced skeletal muscle insulin resistance, mitochondrial function and ectopic lipid accumulation[34, 35].

Thioredoxin-interacting protein (TXNIP), an a-arrestin family member of signaling proteins, impairs insulin signaling through inhibition of thioredoxin NADPH-reduction of sulfur residues on phosphatidylinositol 3-phosphatase and blunts glucose uptake[36]. A recent study in obese humans, Johnson et al.[37•] used caloric restriction as a model to improve skeletal muscle insulin sensitivity. In the absence of changes in mitochondrial capacity or oxidant emission or intramuscular lipid (and metabolite) content, this study identified decreased TXNIP as a mediator of improvements in insulin sensitivity in this model. Ahn et al.[38••] subsequently found that TXNIP is regulated by MondoA; transcription factor thought to regulate carbon and energy homeostasis, intramyocellular triglyceride synthesis and de novo lipogenesis. Further, Kaadige et al.[39] found that mammalian target of rapamycin (mTOR), a Ser/Thr kinase, along with ROS, regulates MondoA, providing evidence for a link between redox signaling and lipid-mediated insulin resistance in skeletal muscle.

\section{Mitochondrial derived peptides}

Mitochondria contain its own DNA comprised of 37 genes encoding proteins, tRNAs, rRNAs and small RNAs[40]. The mitochondria produce short peptides (also called 'mitochondrial derived peptides') that can act as circulating signaling molecules capable of affecting several areas of health and disease[41]. Humanin, the first mitochondrial derived peptide identified, improves insulin sensitivity, $\beta$ cell survival, and delays the onset to diabetes[42]. Recently, Cobb et al.[43] found an additional six small humanin-like peptides (SHLP 1-6) that improved mitochondrial metabolism, reduced ROS generation, and improved insulin sensitivity. Moreover, a second mitochondria-derived peptide called mitochondrial open reading frame of the $12 \mathrm{~S}$ rRNA-c (MOTS-c) was recently discovered by Lee et al.[44••]. MOTS-c was shown to positively regulate skeletal muscle insulin sensitivity and prevent obesity during high-fat feeding, likely through AMPK activation. These data indicate that the mitochondria might regulate insulin sensitivity and metabolic homeostasis through secreted peptides encoded by their own genome.

\section{Sirtuins}

Sirtuins (SIRT) are NAD+-dependent deacetylases that first gained their notoriety in the aging field however; SIRT3 has been shown to play an important role in skeletal muscle insulin sensitivity through alterations in mitochondrial function, oxidative stress, and insulin signaling[45]. Recently, another sirtuin (SIRT6) has also been shown to regulate mitochondrial function and insulin sensitivity [46]. Specifically, Cui et al. found that SIRT6 deletion decreased expression of genes associated with glucose and lipid uptake, fatty acid oxidation, and mitochondrial function and that overexpression of SIRT6 activated AMPK. Taken together, sirtuins appear to play an important role in mitochondrial function and 
metabolic homeostasis and alterations in SIRT3/6 could contribute to skeletal muscle insulin resistance.

\section{Concluding Remarks}

The role of the mitochondria plays in skeletal muscle insulin resistance has been intensely investigated and debated for over twenty years. Early studies focused on the effects of mitochondrial deficiency (i.e. impaired capacity) on glucose and lipid metabolism but subsequent studies have shown that maximal mitochondrial capacity does not have significant effects on basal mitochondrial metabolism. Other work indicated that specific metabolites/intermediates of ectopic lipid accumulation impedes insulin signaling however not all studies agree on the precise mechanisms of lipid-induced insulin resistance in skeletal muscle. Much of the recent data suggest that mitochondrial signaling, including ROS, redox pathways, acylcarnitines, mitochondrial derived peptides, microRNAs[47] or other novel proteins (e.g. Brca1)[48] play a significant role in mitochondrial function, including the regulation of both lipid and glucose metabolism. In addition, it is apparent that mitochondrial bioenergetic state (i.e. energy flux) can significantly influence the interpretation of the relationship between mitochondrial function and metabolic control. In conclusion, it is clear that the role of the mitochondria in substrate metabolism in skeletal muscle is multi-faceted and dependent on bioenergetic state and advances in scientific technology will ultimately demonstrate in greater detail the manifold role of the mitochondria plays in skeletal muscle insulin resistance.

\section{Financial support and sponsorship}

This work was supported by National Institutes of Health grants HL107406-01A1, DK 56341 (Nutrition and Obesity Research Unit at Washington University), and UL1 RR024992 (Washington University Clinical Translational Science Award).

\section{References and recommended reading}

Papers of particular interest, published within the annual period of review, have been highlighted as:

-of special interest

••of outstanding interest

[1]. Centers for Disease Control and Prevention. National Diabetes Statistics Report, 2017: Estimates of Diabetes and Its Burden in the United States.

[2]. Ng JM, Azuma K, Kelley C et al. PET imaging reveals distinctive roles for different regional adipose tissue depots in systemic glucose metabolism in nonobese humans. Am J Physiol Endocrinol Metab 2012; 303:E1134-1141. [PubMed: 22967498]

[3]. Goodpaster BH. Mitochondrial deficiency is associated with insulin resistance. Diabetes 2013; 62:1032-1035. [PubMed: 23520282]

[4]. Holloszy JO. "Deficiency" of mitochondria in muscle does not cause insulin resistance. Diabetes 2013; 62:1036-1040. [PubMed: 23520283]

[5]. Nunnari J, Suomalainen A. Mitochondria: in sickness and in health. Cell 2012; 148:1145-1159. [PubMed: 22424226] 
[6]. Randle PJ, Garland PB, Hales CN, Newsholme EA. The glucose fatty-acid cycle. Its role in insulin sensitivity and the metabolic disturbances of diabetes mellitus. Lancet 1963; 1:785-789. [PubMed: 13990765]

[7]. Samuel VT, Shulman GI. Mechanisms for insulin resistance: common threads and missing links. Cell 2012; 148:852-871. [PubMed: 22385956]

[8]. Rachek LI. Free fatty acids and skeletal muscle insulin resistance. Prog Mol Biol Transl Sci 2014; 121:267-292. [PubMed: 24373240]

[9]. Eldor R, Norton L, Fourcaudot M et al. Increased lipid availability for three days reduces whole body glucose uptake, impairs muscle mitochondrial function and initiates opposing effects on PGC-1alpha promoter methylation in healthy subjects. PloS One 2017; 12:e0188208. [PubMed: 29261667]

[10]. Goodpaster BH, Sparks LM. Metabolic Flexibility in Health and Disease. Cell Metab 2017; 25:1027-1036. [PubMed: 28467922]

[11]. Louche K, Badin PM, Montastier E et al. Endurance exercise training up-regulates lipolytic proteins and reduces triglyceride content in skeletal muscle of obese subjects. J Clin Endocrinol Metab 2013; 98:4863-4871. [PubMed: 24178794]

[12]. Seghieri M, Trico D, Natali A. The impact of triglycerides on glucose tolerance: Lipotoxicity revisited. Diabetes Metab 2017; 43:314-322. [PubMed: 28693962]

[13]. Muoio DM, Neufer PD. Lipid-induced mitochondrial stress and insulin action in muscle. Cell Metab 2012; 15:595-605. [PubMed: 22560212]

[14]. Fisher-Wellman KH, Weber TM, Cathey BL et al. Mitochondrial respiratory capacity and content are normal in young insulin-resistant obese humans. Diabetes 2014; 63:132-141. [PubMed: 23974920]

[15]. Goodpaster BH, He J, Watkins S, Kelley DE. Skeletal muscle lipid content and insulin resistance: evidence for a paradox in endurance-trained athletes. J Clin Endocrinol Metab 2001; 86:57555761. [PubMed: 11739435]

[16]. Burkart AM, Tan K, Warren L et al. Insulin Resistance in Human iPS Cells Reduces Mitochondrial Size and Function. Scientific Rep 2016; 6:22788.

[17]. Fisher-Wellman KH, Neufer PD. Linking mitochondrial bioenergetics to insulin resistance via redox biology. Trends Endocrinol Metab 2012; 23:142-153. [PubMed: 22305519]

[18]. Sparks LM, Gemmink A, Phielix E et al. ANT1-mediated fatty acid-induced uncoupling as a target for improving myocellular insulin sensitivity. Diabetologia 2016; 59:1030-1039. [PubMed: 26886198] - This study used humans and animal and cell models to demonstrate the role of the mitochondrial protein adenine nucleotide translocase 1 (ANT1) in fatty-acid induced uncoupling and insulin sensitivity. Trained humans had higher expression of ANT1, better insulin sensitivity and more uncoupling. Zucker diabetic fatty rats had lower uncoupling and insulin resistance and C2C12 myotubes with partial knockdown of ANT1 had lower sensitivity to uncoupling and insulin-stimulated glucose uptake.

[19]. Morrow RM, Picard M, Derbeneva O et al. Mitochondrial energy deficiency leads to hyperproliferation of skeletal muscle mitochondria and enhanced insulin sensitivity. PNAS 2017; 114:2705-2710. [PubMed: 28223503] • • This study used a knockout model of ANT1 to demonstrate the role of ANT1 in mitochondrial associated insulin resistance. Knockout of ANT1 resulted in partial mitochondrial uncoupling, higher respiration, higher insulin sensitivity and resistance to a high fat diet.

[20]. Muoio DM, Noland RC, Kovalik JP et al. Muscle-specific deletion of carnitine acetyltransferase compromises glucose tolerance and metabolic flexibility. Cell Metab 2012; 15:764-777. [PubMed: 22560225]

[21]. Aguer C, McCoin CS, Knotts TA et al. Acylcarnitines: potential implications for skeletal muscle insulin resistance. FASEB J 2015; 29:336-345. [PubMed: 25342132]

[22]. Liepinsh E, Makrecka-Kuka M, Makarova E et al. Decreased acylcarnitine content improves insulin sensitivity in experimental mice models of insulin resistance. Pharmacol Res 2016; 113:788-795. [PubMed: 26621248]

[23]. Sun L, Liang L, Gao X et al. Early Prediction of Developing Type 2 Diabetes by Plasma Acylcarnitines: A Population-Based Study. Diabetes care 2016; 39:1563-1570. [PubMed: 
27388475] • -In a large human study of 2,103 Chinese subjects, elevated plasma long-chain acylcarnitines increased the risk of the development of type 2 diabetes 2-9 fold.

[24]. Batchuluun B, Al Rijjal D, Prentice KJ et al. Elevated Medium Chain-Acylcarnitines are Associated with Gestational Diabetes, and Early Progression to Type-2 Diabetes, and Induce Pancreatic beta-Cell Dysfunction. Diabetes 2018.

[25]. Consitt LA, Koves TR, Muoio DM et al. Plasma acylcarnitines during insulin stimulation in humans are reflective of age-related metabolic dysfunction. Biochem Biophys Res Commun 2016; 479:868-874. [PubMed: 27693789]

[26]. Di Meo S, Iossa S, Venditti P. Skeletal muscle insulin resistance: role of mitochondria and other ROS sources. J Endocrinol 2017; 233:R15-R42. [PubMed: 28232636]

[27]. Henriksen EJ. Effects of $\mathrm{H} 2 \mathrm{O} 2$ on insulin signaling the glucose transport system in mammalian skeletal muscle. Methods Enzymol 2013; 528:269-278. [PubMed: 23849871]

[28]. Anderson EJ, Lustig ME, Boyle KE et al. Mitochondrial H2O2 emission and cellular redox state link excess fat intake to insulin resistance in both rodents and humans. The J Clin Invest 2009; 119:573-581. [PubMed: 19188683]

[29]. Konopka AR, Asante A, Lanza IR et al. Defects in mitochondrial efficiency and $\mathrm{H} 2 \mathrm{O} 2$ emissions in obese women are restored to a lean phenotype with aerobic exercise training. Diabetes 2015; 64:2104-2115. [PubMed: 25605809]

[30]. Lee HY, Lee JS, Alves T et al. Mitochondrial-Targeted Catalase Protects Against High-Fat DietInduced Muscle Insulin Resistance by Decreasing Intramuscular Lipid Accumulation. Diabetes 2017; 66:2072-2081. [PubMed: 28476930]

[31]. Jones DP, Sies H. The Redox Code. Antioxid Redox Signal 2015; 23:734-746. [PubMed: 25891126]

[32]. Kuban-Jankowska A, Gorska M, Knap N et al. Protein tyrosine phosphatases in pathological process. Front Biosci (Landmark Ed) 2015; 20:377-388. [PubMed: 25553456]

[33]. Copps KD, White MF. Regulation of insulin sensitivity by serine/threonine phosphorylation of insulin receptor substrate proteins IRS1 and IRS2. Diabetologia 2012; 55:2565-2582. [PubMed: 22869320]

[34]. Amrutkar M, Cansby E, Chursa U et al. Genetic Disruption of Protein Kinase STK25 Ameliorates Metabolic Defects in a Diet-Induced Type 2 Diabetes Model. Diabetes 2015; 64:2791-2804. [PubMed: 25845663]

[35]. Chursa U, Nunez-Duran E, Cansby E et al. Overexpression of protein kinase STK25 in mice exacerbates ectopic lipid accumulation, mitochondrial dysfunction and insulin resistance in skeletal muscle. Diabetologia 2017; 60:553-567. [PubMed: 27981357]

[36]. Alhawiti NM, Al Mahri S, Aziz MA et al. TXNIP in Metabolic Regulation: Physiological Role and Therapeutic Outlook. Curr Drug Targets 2017; 18:1095-1103. [PubMed: 28137209]

[37]. J Johnson ML, Distelmaier K, Lanza IR et al. Mechanism by Which Caloric Restriction Improves Insulin Sensitivity in Sedentary Obese Adults. Diabetes 2016; 65:74-84. [PubMed: 26324180] -This study used a 16-week caloric restriction model in humans to improve whole-body insulin sensitivity. While caloric restriction has been previously shown to increase insulin sensitivity, this study demonstrated that improvements were made in the absence of changes in mitochondrial capacity, ROS emissions, or lipid metabolite levels, but caloric restriction lowered TXNIP levels, indicating the important role this protein might play in the relationship between mitochondira and insulin resistance in skeletal muscle.

[38]. Ahn B, Soundarapandian MM, Sessions H et al. MondoA coordinately regulates skeletal myocyte lipid homeostasis and insulin signaling. J Clin Invest 2016; 126:3567-3579. [PubMed: 27500491] • This study expanded the findings of Johnson et al. 2016 by identifiying the transcription factor MondoA as a key regulator of TXNIP and subsequent lipid accumulation and insulin sensitivity in rat myocytes and human skeletal muscle myotubes.

[39]. Kaadige MR, Yang J, Wilde BR, Ayer DE. MondoA-Mlx transcriptional activity is limited by mTOR-MondoA interaction. Mol Cell Biol 2015; 35:101-110. [PubMed: 25332233]

[40]. Nicholls TJ, Rorbach J, Minczuk M. Mitochondria: mitochondrial RNA metabolism and human disease. Int J Biochem Cell Biol 2013; 45:845-849. [PubMed: 23333854] 
[41]. Kim SJ, Xiao J, Wan J et al. Mitochondrially derived peptides as novel regulators of metabolism. J Physiol 2017; 595:6613-6621. [PubMed: 28574175]

[42]. Kuliawat R, Klein L, Gong Z et al. Potent humanin analog increases glucose-stimulated insulin secretion through enhanced metabolism in the beta cell. FASEB J 2013; 27:4890-4898. [PubMed: 23995290]

[43]. Cobb LJ, Lee C, Xiao J et al. Naturally occurring mitochondrial-derived peptides are agedependent regulators of apoptosis, insulin sensitivity, and inflammatory markers. Aging (Albany NY) 2016; 8:796-809. [PubMed: 27070352]

[44]. Lee C, Zeng J, Drew BG et al. The mitochondrial-derived peptide MOTS-c promotes metabolic homeostasis and reduces obesity and insulin resistance. Cell Metab 2015; 21:443-454. [PubMed: 25738459]

[45]. Huynh FK, Muoio DM, Hirschey MD. SIRT3 Directs Carbon Traffic in Muscle to Promote Glucose Control. Diabetes 2015; 64:3058-3060. [PubMed: 26294425]

[46]. Cui X, Yao L, Yang X et al. SIRT6 regulates metabolic homeostasis in skeletal muscle through activation of AMPK. Am J Physiol Endocrinol Metab 2017; 313:E493-E505. [PubMed: 28765271]

[47]. Zhang Y, Zhao YP, Gao YF et al. Silencing miR-106b improves palmitic acid-induced mitochondrial dysfunction and insulin resistance in skeletal myocytes. Mol Med Rep 2015; 11:3834-3841. [PubMed: 25529328]

[48]. Jackson KC, Tarpey MD, Valencia AP et al. Induced Cre-mediated knockdown of Brca1 in skeletal muscle reduces mitochondrial respiration and prevents glucose intolerance in adult mice on a high-fat diet. FASEB J 2018:fj201700464R. 


\section{Purpose of review}

The role of mitochondria in the development of skeletal muscle insulin resistance has been an area of intense investigation and debate for over 20 years. The mitochondria is a multi-faceted organelle that plays an integral part in substrate metabolism and cellular signaling. This article aims to summarize the current findings and thought regarding the relationship between mitochondria and insulin resistance in skeletal muscle. 


\section{Recent findings}

Skeletal muscle insulin resistance was earlier thought to result from deficiency in mitochondrial oxidative capacity and ectopic lipid accumulation. Recent evidence suggests that skeletal muscle insulin resistance in high-energy intake models (i.e. obesity) results primarily from disrupted mitochondrial bioenergetics and alterations in mitochondrial-associated cell signaling. These signaling pathways include reactive oxygen species and redox balance, fatty acid $\beta$-oxidation intermediates, mitochondrial derived peptides, sirtuins, microRNAs and novel nuclear-encoded, mitochondria-acting peptides. 


\section{Key Points}

- $\quad$ Mitochondrial 'deficiency' (i.e. reduced maximal oxidative capacity) does not appear to mediate ectopic skeletal muscle lipid accumulation and insulin resistance.

- Mitochondrial bioenergetic state (i.e. energy flux) can significantly influence the relationship between mitochondrial function and metabolic control.

- Recent data suggest that mitochondrial signaling, including ROS, redox pathways, acylcarnitines, mitochondrial derived peptides, microRNAs or other novel proteins play a significant role in mitochondrial function, including the regulation of both lipid and glucose metabolism. 